\title{
Cotidiano: um software para auxiliar crianças autistas em suas atividades diárias
}

\author{
Afranio F. O. Neto' ${ }^{1}$, Hugo Leonardo P. Rufino², Paula T. Nakamoto ${ }^{3}$, Rodolfo B. \\ Palis $^{4}$, Diovane G. Beira ${ }^{5}$ \\ 1,2,3,4,5 Instituto Federal do Triângulo Mineiro (IFTM) - Uberaba, MG - Brasil. \\ afranio.oliveira@ifms.edu.br, \{hugo, paula, rodolfopalis\}@iftm.edu.br, \\ professor.diovane@hotmail.com
}

\begin{abstract}
Autism is a disorder that presents disorders which cause difficulties in social relations, communication problems and abrupt changes in children behavior. This search observed that there are technologies which help the autism treatment. Nevertheless, it was noticed there is software shortage available to highlight this issues. Therefore, a study was carried out to develop an application to help autistic children in their daily lives. It was tested by a multidisciplinary team, who gave positive feedback that demonstrate how important this tools are to improve the autistic children lives, as well as their interpersonal skills.
\end{abstract}

Resumo. $O$ autismo é um transtorno que apresenta perturbações que causa dificuldade nas relações sociais, problemas de comunicação e alterações bruscas de comportamento. Este estudo averiguou as tecnologias existentes que auxiliam no tratamento do autismo e constatou que existe uma escassez de softwares que levam em consideração esse assunto. Diante disso, foi realizado um estudo para o desenvolvimento de um aplicativo para auxiliar crianças autistas em seu cotidiano. $O$ mesmo foi testado por uma equipe multidisciplinar, que deram um feedback positivo, no sentido que o aplicativo poderá contribuir na vida de uma criança autista de nível leve para moderado, melhorando suas relações sociais.

\section{Introdução}

A sociedade, a partir do século XIX, tem buscado formas de quebrar o paradigma cartesiano, com padrões pré-estabelecidos, em que aqueles que não se adequam a ele, se sentem excluídos. A tendência é de fazer com que todas as pessoas, principalmente aquelas excluídas, tenham formas de se inserirem na sociedade como um todo e se sentirem aceitas. Muitas vezes, profissionais e a família ainda não sabem a melhor forma de colaborar com crianças que possuem necessidades específicas, em especial o autismo, foco desta pesquisa. Isto ocorre, principalmente, por essa síndrome ser mal compreendida, tendo apenas de 10 a $15 \%$ dos casos com causa genética específica. Mas essa síndrome desafia os pesquisadores que buscam, não somente tratamentos, mas soluções definitivas (GOMES, 2014). Diante de tal situação, torna-se importante conhecer as especificidades do transtorno e, assim, poder intervir, educacionalmente, de forma mais adequada. 
O autismo é uma síndrome do comportamento humano, descoberta por Kanner (1997), no final da década de 1930; essa síndrome se caracterizou pela dificuldade das pessoas em manterem relações sociais, desde a infância, por deficiência de comunicação e linguagem, não conseguindo aceitar a mudança de rotina e no aprendizado. Apesar dessa síndrome não possuir causas definidas e nem cura definitiva, ela pode ser diagnosticada precocemente e tratada para que as pessoas portadoras tenham uma melhor qualidade de vida. Há diferentes visões a respeito do autismo, mas o que se sabe é que não se separa o desenvolvimento cognitivo do desenvolvimento afetivo. Portanto torna-se fundamental conhecer as formas de abordagens de intervenções pedagógicas: ABA (Análise Aplicada do Comportamento), PECS (Sistema de Comunicação por meio de figuras), TEACCH (Programa de aprendizado individualizado), para serem aplicadas às crianças autistas.

Por conseguinte, buscou-se alguma tecnologia de apoio, denominada tecnologia assistiva, que permitisse a utilização de intervenções pedagógicas para auxiliar no tratamento do autismo. Durante a busca foi possível observar que existem muitas tecnologias utilizadas para ensino de crianças, porém foram encontradas um número menor de tecnologias desenvolvidas com foco em crianças autistas.

O objetivo geral deste trabalho é desenvolver uma proposta para criação do aplicativo para dispositivos móveis a ser aplicado no cotidiano de crianças com autismo, nos níveis leve e moderado.

A presente pesquisa se justifica pelo desejo de utilizar a tecnologia para dar mais autonomia e inclusão social para pessoas que tenham algum tipo de deficiência. Neste contexto, foram pesquisados vários tipos de deficiências: Físicas, Visuais e Neurológicas, bem como os recursos tecnológicos que já existem com intuito de auxiliar as pessoas supracitadas. Por meio das informações levantadas na internet, pôde-se notar que existe um número maior de intervenções, utilizando tecnologias para pessoas com deficiência física, do que para pessoas com deficiências neurológicas. Esse cenário encontrado contribuiu para que fosse definido que o trabalho iria vislumbrar $o$ tratamento de uma deficiência neurológica. Por conseguinte, foi realizada uma investigação sobre algumas deficiências neurológicas, onde uma delas chamou a atenção, o autismo, por estar associada a crianças e apresentar uma evolução significativa do número de casos dessa patologia. Esses fatos motivaram $o$ desenvolvimento de um aplicativo móvel para tratamento do autismo.

\section{Fundamentação teórica}

O objetivo deste capítulo é fornecer o embasamento teórico e a contextualização do autismo, intervenções educacionais, tecnologias para o tratamento dessa patologia e as técnicas e tecnologias utilizadas para o desenvolvimento deste trabalho.

\section{1 - Autismo}

A primeira publicação sobre autismo foi feita por Leo Kanner, em 1943, ele escreveu o histórico artigo: "Os distúrbios autísticos de contato afetivo". Neste artigo, Kanner descreveu o estudo feito com crianças em idade inferior a 11 anos, que 
apresentavam comportamentos diferentes e singulares de outras crianças observadas, até então. Foi observado nessa pesquisa que, apesar das diferenças individuais, existiam características comuns, essenciais e inevitáveis. A principal característica constatada foi que essas crianças tinham uma inabilidade no relacionamento interpessoal, que as distinguia de outras patologias como a esquizofrenia: "o distúrbio fundamental mais surpreendente é a incapacidade dessas crianças de estabelecer relações de maneira normal com as pessoas e situações, desde o princípio de suas vidas". [Kanner 1997]. Em 1944, o também austríaco Hans Asperger, escreveu um artigo em alemão: "Psicopatologia autística da infância", que descreveu características apresentadas por crianças, bem semelhantes às descritas por Kanner. [Asperger 1994]. Esses autores foram os precursores da identificação do autismo e, posteriormente, foram estudados por outros pesquisadores.

O autismo, há décadas, foi considerado uma condição relativamente rara, mas, dados epidemiológicos têm alterado radicalmente essa percepção. Com base em uma ampla pesquisa realizada pelo CDC (Centro de Controle e prevenção de doenças), nos EUA, estimou a ocorrência de autismo em 1 a cada 88 crianças, com maior frequência (5 vezes) em crianças do sexo masculino: 1 a cada 54. E, no sexo feminino, 1 a cada 252. No entanto, estudos epidemiológicos são difíceis de comparar, pois há diferenças na população pesquisada, nos mecanismos de recrutamento, tamanho da amostra, critérios de diagnósticos, instrumentos utilizados, bem como se critérios de imparidade são incluídos [Fombonne 2009]. Mesmo assim, utilizando a mesma metodologia ao longo de um período de oito anos, foi criada uma rede de monitoramento que encontrou aumento nas taxas de autismo nos EUA, conforme apresentado na (Tabela 1).

Tabela 1: Identificar prevalência de transtornos do espectro do autismo

\begin{tabular}{|c|c|c|c|c|}
\hline \multicolumn{5}{|c|}{$\begin{array}{c}\text { Identified Prevalence of Autism Spectrum Disorder } \\
\text { ADDM Network 2000-2010 } \\
\text { Combining Data from All Sites }\end{array}$} \\
\hline Surveillance Year & Birth Year & $\begin{array}{l}\text { Number of ADDM } \\
\text { Sites Reporting }\end{array}$ & $\begin{array}{l}\text { Prevalence per } \\
1,000 \text { Children } \\
\text { (Range) }\end{array}$ & $\begin{array}{l}\text { This is about } \\
1 \text { in } X \text { children... }\end{array}$ \\
\hline 2000 & 1992 & 6 & $\begin{array}{c}6.7 \\
(4.5-9.9)\end{array}$ & 1 in 150 \\
\hline 2002 & 1994 & 14 & $\begin{array}{c}6.6 \\
(3.3-10.6)\end{array}$ & 1 in 150 \\
\hline 2004 & 1996 & 8 & $\begin{array}{c}8.0 \\
(4.6-9.8)\end{array}$ & 1 in 125 \\
\hline 2006 & 1998 & 11 & $\begin{array}{c}9.0 \\
(4.2-12.1)\end{array}$ & 1 in 110 \\
\hline 2008 & 2000 & 14 & $\begin{array}{c}11.3 \\
(4.8-21.2)\end{array}$ & 1 in 88 \\
\hline 2010 & 2002 & 11 & $\begin{array}{c}14.7 \\
(5.7-21.9)\end{array}$ & 1 in 68 \\
\hline
\end{tabular}

Fonte: CDC,2016

São várias as possíveis causas do autismo, porém as mais aceitas estão ligadas a neurociência, que pesquisa sobre anormalidades em alguma parte do cérebro humano, porém ainda não se conseguiu definir de forma conclusiva. 
Por não existir marcadores biológicos, o diagnóstico do autista é ainda baseado em critérios comportamentais, mesmo que as pesquisas sobre o tema estejam em constante avanço, ainda há uma distância muito grande na compreensão desse fenômeno [Moura et al 2005 p. 112].

Mesmo assim, estas evidências apontam para a melhor forma de diagnosticar o autismo, que pode se manifestar precocemente por meio de perturbações, geralmente antes dos três anos de idade.

\section{2 - Perturbações}

Lorna Wing (1979) separa as perturbações que as crianças autistas possuem em três diferentes áreas de domínio: 1. Social; 2. Linguagem e Comunicação; 3. Comportamento e Pensamento. Todavia Baptista \& Bosa salientam que, apesar de Lorna Wing separar as três perturbações (tríade), elas não são separáveis, como leva a crer o termo "tríade", "a expressão resultou de mensurações estatísticas, demonstrando que os comprometimentos que apareciam nessas áreas não ocorriam ao acaso; apresentavam-se juntos, embora com intensidade e qualidades variadas" (Baptista and Bosa 2002 p.34].

\section{3 - Níveis de Autismo}

Os níveis de autismo são definidos pela DSM (Diagnostic and Statistical Manual of Mental Disorders), que é um guia publicado pela Associação Psiquiátrica Americana, no qual os médicos se baseiam para fornecer um diagnóstico formal [Frazier 2012]. A DSM-5, trouxe uma mudança em relação as edições anteriores, onde foi criado o termo TEA (Transtorno do Espectro do Autismo), que engloba todos os tipos de sintomas autísticos. E, para diferenciar esses sintomas, foram criados três níveis: leve, moderado e severo, que são analisados por meio da gravidade, baseados na necessidade do apoio destinado, devido aos desafios com a comunicação social, interesses restritos e comportamentos repetitivos. Porém, há uma grande heterogeneidade na apresentação fenotípica do TEA, tanto com relação à configuração e severidade dos sintomas comportamentais. [Geschwind 2009]

O autismo é um distúrbio, ainda, incurável, mesmo nos níveis mais leves, porém se a criança for diagnosticada nos primeiros anos de vida e houver um estímulo por parte da família, poderá obter ganhos significativos, principalmente nos casos de menor deficiência mental. [Schwartzman 2011]

\section{4 - Intervenções Educacionais}

Esse tópico irá abordar os principais tipos de intervenções educacionais (métodos de aprendizagem) que auxiliam os pais e os educadores, a desenvolverem atividades com crianças autistas.

A ABA (Análise Aplicada ao Comportamento) é um tratamento comportamental, que objetiva ensinar habilidades que a crianças não as detêm, por meio 
de fases bem definidas, pois o autista tem grande dificuldade em generalizar estímulos. Essas habilidades são ensinadas, geralmente, de maneira individualizada, onde a criança autista aprende por meio de instruções e, quando ela consegue realizar determinada atividade, ela recebe um estimulo, o que torna a atividade prazerosa. Inicialmente, são ensinadas habilidades básicas como: realizar o contato visual, sentar, esperar sua vez, para depois partir para as situações de grupo e pedagógicas. [Mello 2007].

O PECS (Sistema de Comunicação por troca de Figuras) é um método considerado simples e de baixo custo, que tem o objetivo de organizar a comunicação por meio de cartões, que funcionam, tanto para linguagens verbais quanto para as nãoverbais. Esse método, apesar de simples, quando bem implementado, produz resultados inquestionáveis, levando o autista a conseguir expressar de forma espontânea, seus desejos, além de melhorar a interação social. [Sampaio 2005].

O último método a ser exposto é o TEACCH (Tratamento e Educação para Crianças Autistas e com Distúrbios na Comunicação), que é um dos mais utilizados, por fazer uma organização do ambiente físico, deixando a criança autista mais segura e confortável. Esse método foi proposto, pois foi observado que quando um autista é levado para um ambiente desconhecido, causa inquietação, irritação e até agressividade. Isso tudo acontece quando a situação foge do habitual [Fonseca 2016].

Esses métodos não são excludentes e sim complementares e auxiliam o autista a adquirir habilidade por meio de rotinas na sua vida diária. Segundo Nilsson (2004, p.5253), "O autista apresenta um pensamento literal concreto, visual, fragmentado", isso mostra a importância da utilização dos métodos apresentados, visto que todos têm natureza visual. Portanto, oferecer uma ferramenta visual e rotineira a uma criança com autismo, além de apresentar as informações de maneira mais compreensíveis, irá estimular o desenvolvimento da aprendizagem.

\section{5 - Tecnologias envolvidas}

Para se definir as tecnologias a serem aplicadas ao projeto, utilizou-se os conceitos de Engenharia de Software, que é a área do conhecimento da engenharia que abrange todos os aspectos da produção de software, desde os estágios iniciais de especificação do sistema até a manutenção do mesmo. Com isso buscou-se uma tecnologia de apoio, denominada de tecnologia assistiva, que ajuda um indivíduo com deficiência, em suas atividades diárias. Há relatos de pessoas com deficiência que utilizaram essa tecnologia, se tornaram e permaneceram independentes. [Cook e Polgar 2015].

Dentre os equipamentos de tecnologia assistiva, uma que se destaca é a utilização de dispositivos móveis, por serem tecnologias com muita disponibilidade no mercado mundial.

O protótipo foi desenvolvido levando em consideração conceitos de usabilidade

A usabilidade é um atributo de qualidade e refere-se à facilidade com que o usuário interage com a interface, ou seja, a rapidez na aprendizagem, a eficiência com que este utiliza os recursos 
disponíveis para executar uma determinada tarefa, o quanto lembram de como usar um recurso já conhecido, a propensão à erros e a motivação a usar os recursos disponíveis novamente, concluindo que se alguns recursos não puderem ou não forem utilizados podem muito bem não existirem. [Nielsen e Loranger 2007 p. 16]

A usabilidade está inserida dentro da área chamada interação homemcomputador e é multidisciplinar, pois suas avaliações e métodos são apoiados por diversas disciplinas como: Psicologia, Economia, Ciência da Computação, dentre outros.

Para documentar o aplicativo foi utilizado os diagramas de Casos de Uso e de Atividades da linguagem Unified Modeling Language - UML, que é uma linguagem visual de modelagem de programas com o paradigma de programação orientado a objetos. Ela fornece uma boa apresentação para o usuário de como será desenvolvido o software por meio de detalhes estruturais minuciosos e aspectos comportamentais de um único sistema, fornecendo vários pontos de vistas, para que o usuário possa ter uma ideia de como funcionará o software antes de sua implementação [Larman 2007].

\section{Metodologia}

As abordagens modernas de sistemas adotam a filosofia baseada em modelos. $\mathrm{O}$ que varia bastante entre os métodos de um sistema, são os tipos de modelos que devem ser construídos, como devem ser construídos e quem deve construí-los. Por esse motivo, com a utilização dos conceitos de Engenharia de Software para produção do aplicativo, foi escolhido o Modelo Cascata, demonstrado na (Figura 1), por ser um método que deixa cada etapa do desenvolvimento bem definidas, o que contribui para um ganho substancial de tempo. Neste modelo, consideram-se as atividades de especificação, desenvolvimento, validação e evolução, fundamentais ao processo e as representam como fases separadas do mesmo: a especificação de requisitos, o projeto de software, a implementação, os testes e, assim por diante. Seu nome é devido à sequência em cascata de uma fase para outra [Sommerville 2004].

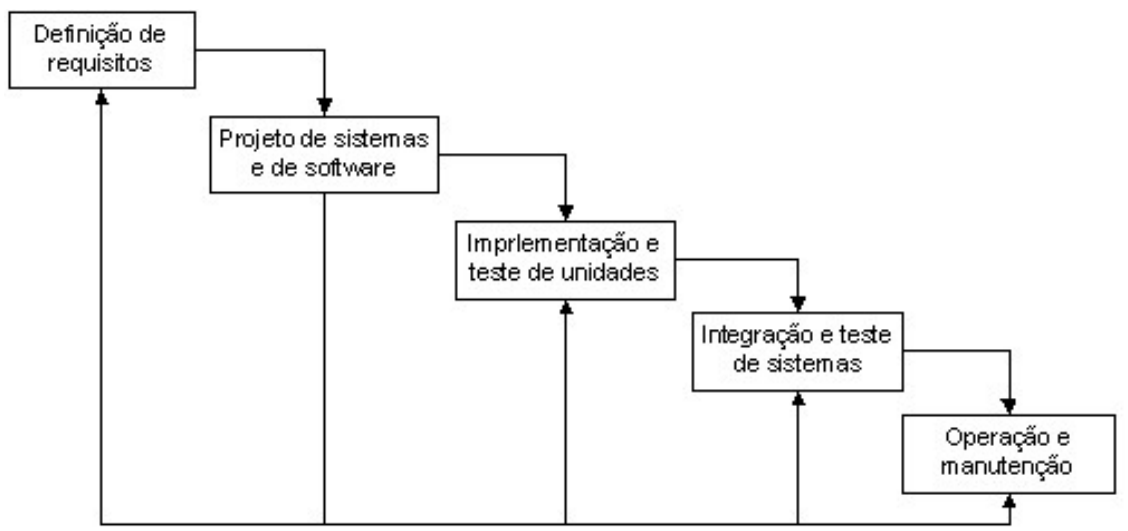

Figura 1: Modelo Cascata

Fonte: SOMMERVILLE, 2004, p.38 
VI Congresso Brasileiro de Informática na Educação (CBIE 2017)

Anais do XXVIII Simpósio Brasileiro de Informática na Educação (SBIE 2017)

Segundo a metodologia de desenvolvimento em cascata, inicialmente, foi realizada a Definição dos requisitos, que consistiu em uma pesquisa qualitativa descritiva e exploratória utilizando métodos de pesquisa científica tecnológica, que iniciou com a busca por cursos sobre autismo e posteriormente a busca de profissionais que trabalham no tratamento do autismo. Esses profissionais foram encontrados e logo foi montado uma equipe multidisciplinar, que contribuíram para a pesquisa por meio de entrevistas e reuniões, que resultaram na definição de um aplicativo para crianças autistas de níveis leve e moderado, que tem como objetivo auxiliar os pais dessas crianças a regularem suas atividades diárias, utilizando como intervenções pedagógicas, a agenda do TEACCH e o conceito de cartões do PEC. Com isso foram levantados e definidos os principais requisitos que o aplicativo iria contemplar, logo foi feita a elaboração da documentação do sistema levando em consideração toda a análise realizada na etapa anterior. Esta documentação foi modelada por meio dos diagramas da UML, e apresentada a equipe multidisciplinar, para que esses diagramas pudessem passar por reajustes antes do início do desenvolvimento, a (Figura 2) apresenta o diagrama de Caso de Uso do Aplicativo.

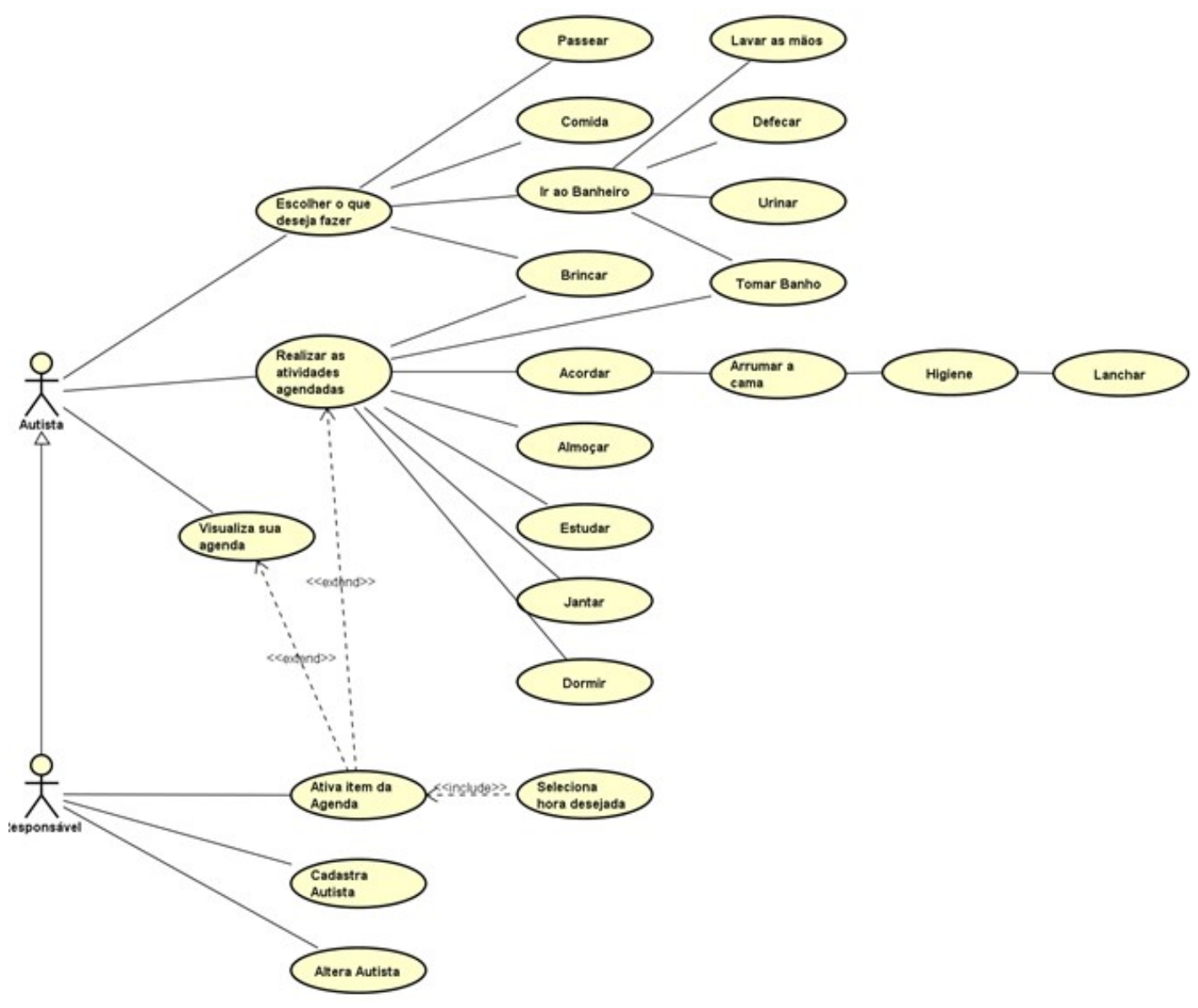

Figura 2: Diagrama de Casos de Uso do Aplicativo Fonte: Dados do próprio trabalho 
VI Congresso Brasileiro de Informática na Educação (CBIE 2017)

Anais do XXVIII Simpósio Brasileiro de Informática na Educação (SBIE 2017)

$\mathrm{Na}$ etapa, Projeto de sistema de software, forma definidas a estruturação dos dados, a arquitetura do software e a caracterização da interface. Com as etapas anteriores completadas, passou-se para a próxima etapa: Implementação e testes de unidade, onde foi desenvolvido um protótipo do aplicativo, que é uma representação do sistema com algumas funcionalidades básicas; este desenvolvimento foi realizado levando em consideração os conceitos de usabilidade, o protótipo passará por simples testes sistêmicos e adequações sugeridas pela equipe como: colocação de limites visuais, colocação de áudio motivacional a cada acerto, não permitir arrastar a sequência errada e a disposição dos componentes na tela, para a utilização ser mais sugestiva. Chegou-se então a uma versão de apresentação observada na (Figura 3), que foi utilizada pela equipe, onde as envolvidas analisaram a efetividade do protótipo, e deram um feedback, dizendo que o aplicativo superou suas expectativas, mostrando-se um aplicativo intuitivo, que seguiu todas as orientações passadas, e que o mesmo poderá ser colocado em testes com crianças autistas de nível leve e moderado. Este feedback, foi conseguido, após ser disponibilizado o aplicativo para a equipe testar durante uma semana, ao final dessa semana foi realizada uma reunião, onde as mesmas mostraram seus pontos de vista.

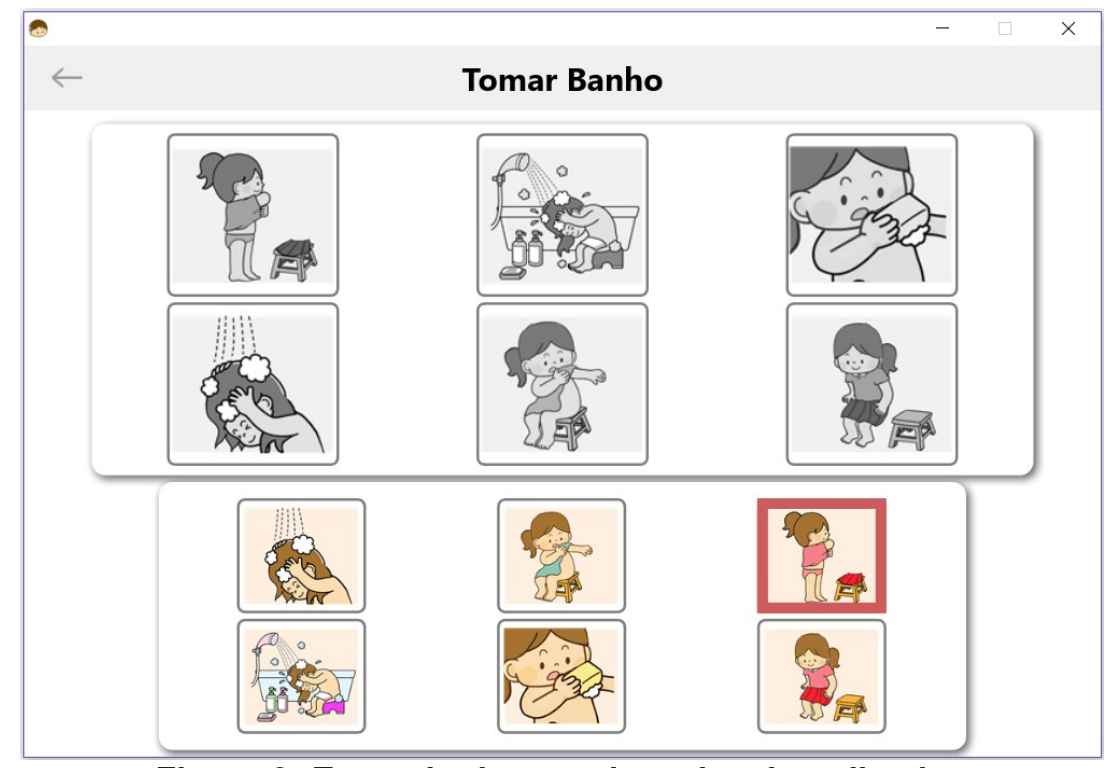

Figura 3: Exemplo de uma das telas do aplicativo Fonte: Dados do próprio trabalho.

\section{Considerações Finais}

Até onde a pesquisa foi realizada, notou-se uma necessidade enorme de aplicativos direcionados a exercícios para crianças autistas. Por sua demonstração das atividades e ferramentas aplicadas a seus clientes, percebe-se que, realmente, há necessidade de maiores pesquisas na área de aplicativos em dispositivos móveis (principalmente, por ser mais cômodo para a criança e para a família) e outras ferramentas que possam colaborar para o desenvolvimento da criança para promover a inclusão. Pela pesquisa bibliográfica, pode ver que é um grande desafio para a família e 
VI Congresso Brasileiro de Informática na Educação (CBIE 2017)

Anais do XXVIII Simpósio Brasileiro de Informática na Educação (SBIE 2017)

para os profissionais, uma vez que a perturbação é bastante complexa, exigindo dos profissionais muita pesquisa e criação de ferramentas adequadas ao autismo.

A pesquisa está com o protótipo pronto com muitas funcionalidades, porém ainda necessita de ser testada com crianças autistas e passar por ajustes para se ter uma versão final, para ser colocado à disposição para download. Mas o protótipo foi testado e muito aceito pela equipe multidisciplinar que contribuiu para a elaboração do aplicativo, a equipe se surpreendeu com os detalhes das intervenções pedagógicas TEACCH e PEC apresentadas no protótipo do aplicativo, deixando um feedback satisfatório sobre a efetividade que poderá ter o aplicativo se utilizado por crianças autistas

Espera-se que, ao ser utilizado por crianças autistas, o aplicativo possa contribuir significativamente para o tratamento dessas crianças, melhorando a qualidade de vida e contribuindo para a inclusão social.

\section{Referências}

Asperger, A. Autistic psychopathy in childhood. In U. Firth (Ed), Autism and Asperger syndrome (pp.37-92). Cambridge: Cambridge University Press, 1994.

Baptista, Cláudio Roberto; BOSA Cleonice; e colaboradores. Autismo e educação: reflexões e propostas de intervenção. Porto Alegre, Artmed, 2002.

CDC. Centers for Disease Control and Prevention. Disponível em:< http://www.cdc.gov/ncbddd/autism/data.html > Acesso: 29 fev 2016.

Cook, Albert M.; POLGAR, Janice M. Assistive Technologies: Principles and Practice, 4 ed. Mosby: Year book, 2015

Fombonne E. Epidemiology of pervasive developmental disorders. Pediatric Research, 65:591-598.2009.

Fonseca, Maria Elisa Granchi; CIOTA, Juliana De Cássia Baptistella. Vejo e Aprendo Fundamentos do Programa Teacch - o Ensino Estruturado Para Pessoas Com Autismo. Ribeirão Preto: Booktoy. 136p, 2016.

Frazier T, Youngstrom E, Speer L. Validation of proposed DSM-5 criteria for autism spectrum disorder. Journal of the American Academy of Child and Adolescent Psychiatry, 51:28-40.2012.

Geschwind, D.H. Advances in Autism. Annual Review of Medicine, v. 60, p. 367-380, 2009.

Gomes, Marina. Biologia do Autismo. Ciência e Cultura. Vol. 66 n. 1. São Paulo, 2014. Disponível em: $<\quad$ http://cienciaecultura.bvs.br/scielo.php?pid=S000967252014000100004\&script=sci_arttext $>$ Acesso: 5 de maio 2016.

Kanner, L. Os distúrbios autísticos de contato afetivo. In: ROCHA, P. S. Autismos. São Paulo: Escuta, 1997. 
VI Congresso Brasileiro de Informática na Educação (CBIE 2017)

Anais do XXVIII Simpósio Brasileiro de Informática na Educação (SBIE 2017)

Larman, C. Utilizando UML e padrões: uma introdução à análise e ao projeto orientados a objetos e ao desenvolvimento iterativo. 1. ed. São Paulo: Bookman, 2007.

Mello, Ana Maria S. Rios. Autismo: guia prático. $7^{\mathrm{a}}$ ed. São Paulo: AMA; Brasília: Corde, 2007

Moura, Paula J.; Sato, Fabio; Mercadante, Marcos T. Bases Neurobiológicas do Autismo: Enfoque no domínio da sociabilidade in Caderno de Pós-graduação em Distúrbios do Desenvolvimento. 2005. Disponível em: $<$ http://www.mackenzie.br/fileadmin/Pos_Graduacao/Mestrado/Disturbios_do_Dese nvolvimento/Publicacoes/volume_IV/000.pdf> Acesso: 8 nov 2016.

Nilsson, Inger. Introdução a educação especial para pessoas com transtornos de espectro autístico e dificuldades semelhantes de aprendizagem. Em PDF.Congresso Nacional sobre a Síndrome de Autismo 2004. Disponível em < http://www.ama.org.br/download/Autismo-IntrodEducEspecial.pdf $>$ Acesso: 23 mar. 2016.

Nielsen, J.; Loranger, H. Usabilidade na Web: Projetando Websites com qualidade. 5. ed. Rio da Janeiro: Elsevier, 2007.

Sampaio, A. S. Transtorno autista e a abordagem cognitivo-comportamental: possibilidade de auxilio psicológico. 2005.Disponível em: www.psicologiavirtual.com.br. Acesso: 29 de out 2015.

Schwartzman, José Salomão; Araújo, Ceres Alves de. Transtornos do espectro do autismo. São Paulo: Memnon, 2011.

Sommerville, Ian. Engenharia de Software. 6.ed. São Paulo: Pearson Education, 2004.

Wing, L., \& Gould, J. (1979). Severe impairments of social interaction and associated abnormalities in children Epidemiology and classification Journal of Autism and Developmental Disorders, 9, 11- 29. 